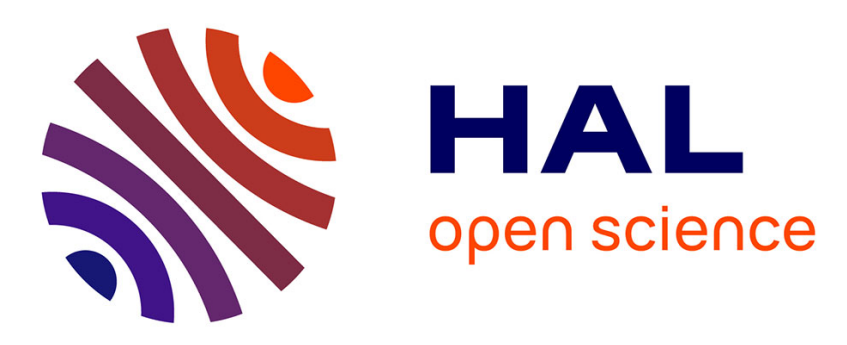

\title{
Extension and its price for the connected vertex cover problem
}

Mehdi Khosravian Ghadikolaei, Nikolaos Melissinos, Jérôme Monnot, Aris T. Pagourtzis

\section{- To cite this version:}

Mehdi Khosravian Ghadikolaei, Nikolaos Melissinos, Jérôme Monnot, Aris T. Pagourtzis. Extension and its price for the connected vertex cover problem. Combinatorial Algorithms - 30th International Workshop, IWOCA 2019, Proceedings, Jul 2019, Pisa, Italy. 10.1007/978-3-030-25005-8 . hal-02344051

\section{HAL Id: hal-02344051 \\ https://hal.science/hal-02344051}

Submitted on 3 Nov 2019

HAL is a multi-disciplinary open access archive for the deposit and dissemination of scientific research documents, whether they are published or not. The documents may come from teaching and research institutions in France or abroad, or from public or private research centers.
L'archive ouverte pluridisciplinaire HAL, est destinée au dépôt et à la diffusion de documents scientifiques de niveau recherche, publiés ou non, émanant des établissements d'enseignement et de recherche français ou étrangers, des laboratoires publics ou privés. 


\title{
Extension and its price for the connected vertex cover problem
}

\author{
Mehdi Khosravian Ghadikoalei ${ }^{1}$, Nikolaos Melissinos ${ }^{2}$, Jérôme Monnot $^{1}$, and \\ Aris Pagourtzis ${ }^{2}$ \\ 1 Université Paris-Dauphine, PSL University, CNRS, LAMSADE, 75016 Paris, \\ France, \{mehdi.khosravian-ghadikolaei, jerome.monnot\}@dauphine.fr \\ 2 School of Electrical and Computer Engineering, National Technical University of \\ Athens, Polytechnioupoli, 15780 Zografou, Athens, Greece, \\ \{nikolaosmelissinos@mail.ntua.gr,pagour@cs.ntua.gr\}
}

\begin{abstract}
We consider extension variants of VERTEX COVER and INDEPENDENT SET, following a line of research initiated in [9]. In particular, we study the ExT-CVC and the ExT-NSIS problems: given a graph $G=(V, E)$ and a vertex set $U \subseteq V$, does there exist a minimal connected vertex cover (respectively, a maximal non-separating independent set) $S$, such that $U \subseteq S$ (respectively, $U \supseteq S$ ). We present hardness results for both problems, for certain graph classes such as bipartite, chordal and weakly chordal. To this end we exploit the relation of ExT-CVC to ExT$\mathrm{VC}$, that is, to the extension variant of VERTEx Cover. We also study the Price of Extension ( $P o E$ ), a measure that reflects the distance of a vertex set $U$ to its maximum efficiently computable subset that is extensible to a minimal connected vertex cover, and provide negative and positive results for PoE in general and special graphs.
\end{abstract}

Key words: extension problems, connected vertex cover, upper connected vertex cover, price of extension, special graph classes, approximation algorithms, NP-completeness

\section{Introduction}

We consider the extension variant of the (Minimum) Connected Vertex Cover (Min CVC) problem and its associated price of extension (PoE); we call this variant Extension Connected Vertex Cover problem (ExT-CVC for short). Intuitively, the extension variant of a minimization problem $\Pi$ is the problem of deciding whether a partial solution $U$ for a given instance of $\Pi$ can be extended to a minimal (w.r.t. inclusion) feasible solution for that instance; PoE refers to the maximum size subset of $U$ that can be extended to a minimal feasible solution. A framework for extension problems is developed in [10] where a number of results are given for several hereditary and antihereditary graph problems. Particular complexity results for the extension of graph problems, such as Vertex Cover, Hitting Set, and Dominating Set, are given in [2, $3,6,9,20-22]$. A subset $S \subseteq V$ of a connected graph $G=(V, E)$ is a connected vertex cover ( $C V C$ for short) if $S$ is a vertex cover (i.e., each edge of $G$ is incident 
to at least a vertex of $S$ ) and the subgraph $G[S]$ induced by $S$ is connected. The corresponding optimization problem (Minimum) Connected Vertex Cover (Min CVC) consists in finding a CVC of minimum size $[12,16,17]$. Given a (connected) vertex cover $S$ of a graph $G=(V, E)$, an edge $e \in E$ is private to a vertex $v \in S$ if $v$ is the only vertex of $S$ incident to $e$. Hence, a vertex cover $S$ of $G$ is minimal iff each vertex $v \in S$ has a private edge. A CVC $S$ of $G$ is minimal if for every $v \in S, S \backslash\{v\}$ is either not connected or not a vertex cover.

In this paper we study Extension Connected Vertex Cover (ExtCVC): given a connected graph $G=(V, E)$ together with a subset $U \subseteq V$ of vertices, the goal is to decide whether there exists a minimal (w.r.t. inclusion) CVC of $G$ containing $U$; note that for several instances the answer is negative. In this latter case we are also interested in a new maximization problem where the goal is to find the largest subset of vertices $U^{\prime} \subseteq U$ which can be extended to a minimal feasible solution. This concept is defined as the Price of Extension (PoE) in [9]. For the two extreme cases $U=\emptyset$ and $U=V$, we note that for the former the question is trivial since there always exists a minimal CVC [27], while for the latter $(U=V)$ the problem is equivalent to finding a minimal CVC of maximum size, (called Upper $C V C$ in the paper).

\subsection{Graph definitions and terminology}

Throughout this article, we consider a simple connected undirected graph without loops $G=(V, E)$ on $n=|V|$ vertices and $m=|E|$ edges. Every edge $e \in E$ is denoted as $e=u v$ for $u, v \in V$. For $X \subseteq V, N_{G}(X)=\{v \in V: v x \in E$, for some $x \in X\}$ and $N_{G}[X]=X \cup N_{G}(X)$ denotes the closed neighborhood of $X$. For singleton sets $X=\{x\}$, we simply write $N_{G}(x)$ or $N_{G}[x]$, even omitting $G$ if it is clear from the context; for a subset $X \subset V, N_{X}(v)=N_{G}(v) \cap X$. The number of neighbors of $x$, called degree of $x$, is denoted by $d_{G}(x)=\left|N_{G}(x)\right|$ and the maximum degree of the graph $G$ is denoted by $\Delta(G)=\max _{v \in V} d_{G}(v)$. A leaf is a vertex of degree one, and $V_{l}$ denotes the set of leaves in $G$. For $X \subseteq V$, $G[X]$ denotes the subgraph induced by $X$, that is the subgraph only containing $X$ as vertices and all edges of $G$ with both endpoints in $X$. A connected graph $G=(V, E)$ is biconnected, if for each pair of vertices $x, y$ there is a simple cycle containing both $x$ and $y$, or equivalently, the removal of any vertex maintains connectivity. A cut-set $X \subset V$ is a subset of vertices such that the deletion of $X$ from $G$ strictly increases the number of connected components. A cut-set which is a singleton is called a cut-vertex and a cut-set $X$ is minimal if $\forall x \in X$, $X \backslash\{x\}$ is not a cut-set. Hence, a graph is biconnected iff it is connected and it does not contain any cut-vertex. In this article, $V_{c}(G)$ denotes the set of cutvertices of a graph $G$; we will simply write $V_{c}$ if $G$ is clear from the context. A graph $G=(L \cup R, E)$ is split (resp. bipartite) where the vertex set $L \cup R$ is decomposed into a clique $L$ and an independent set $R$ (resp. two independent sets). A graph is chordal if all its cycles of length at least four have a chord, that is, an edge connecting nonconsecutive vertices of the cycle. There are many characterizations of chordal graphs. One of them, known as Dirac's theorem, asserts that a graph $G$ is chordal iff each minimal cut-set of $G$ is a clique. Recall 
that $S \subseteq V$ is a vertex cover, if for each $e=u v \in E, S \cap\{u, v\} \neq \emptyset$ while $S \subseteq V$ is an independent set if for each pair of vertices $u, v$ of $S, u v \notin E$; $S$ is a vertex cover iff $V \backslash S$ is an independent set of $G=(V, E)$. The minimum vertex cover problem (MIN VC for short) asks to find a vertex cover of minimum size and the maximum independent set problem (MAX IS for short), asks to find an independent set of maximum size for a given graph.

\subsection{Problem definitions}

As mentioned above, we consider the extension variants of two optimization problems: the (Minimum) Connected Vertex Cover problem (Min CVC) and the (Maximum) Non Separating Independent Set problem (MAX NSIS). A non separating independent set $S$ of a connected graph $G=(V, E)$ is a subset of vertices of $G$ which is independent (i.e., any two vertices in $S$ are non adjacent) and $S$ is not a cut-set of $G$. MAx NSIS asks to find a non separating independent set of maximum size. MIN CVC and MAX NSIS have been studied in $[16,12,30,15,26]$ where it is proved that the problems are polynomially solvable in graphs of maximum degree 3, while in graphs of maximum degree 4 they are NP-hard.

\section{ExT-CVC}

Input: A connected graph $G=(V, E)$ and a presolution (also called set of forced vertices) $U \subseteq V$.

Question: Does $G$ have a minimal connnected vertex cover $S$ with $U \subseteq S$ ?

Dealing with ExT-NSIS, the goal to decide the existence of a maximal NSIS excluding vertices from $V \backslash U$.

\section{EXT-NSIS}

Input: A connected graph $G=(V, E)$ and a frontier subset $U \subseteq V$. Question: Does $G$ have a maximal NSIS $S$ with $S \subseteq U$ ?

Considering the possibility that some set $U$ might not be extensible to any minimal solution, one might ask how far is $U$ from an extensible set. This concept, introduced in [9], is called Price of Extension (PoE). This notion is defined in an attempt to understand what effect the additional presolution constraint has on the possibility of finding minimal solutions. A similar approach has already been used in the past under the name the Price of Connectivity in [7] for the context of connectivity because it is a crucial issue in networking applications. This notion has been introduced in [7] for MIN VC and is defined as the maximum ratio between the connected vertex cover number and the vertex cover number. In our context, the goal of PoE is to quantify how close efficiently computable extensible subsets of the given presolution $U$ are to $U$ or to the largest possible extensible subsets of $U$. To formalize this, we define two optimization problems as follows: 
Max EXT-CVC

Input: A connected graph $G=(V, E)$ and a set of vertices $U \subseteq V$.

Feasible Solution: Minimal connected vertex cover $S$ of $G$.

Goal: Maximize $|S \cap U|$.

\section{Min ExT-NSIS}

Input: A connected graph $G=(V, E)$ and a set of vertices $U \subseteq V$.

Feasible Solution: Maximal non separating independent set $S$ of $G$.

Goal: Minimize $|S \cup U|$.

For $\Pi \in\{$ Max Ext-CVC, Min Ext-NSIS $\}$, we denote by $\operatorname{opt}_{\Pi}(G, U)$ the value of an optimal solution. Since for both of them opt $t_{\Pi}(G, U)=|U|$ iff $(G, U)$ is a yes-instance of the extension variant, we deduce that MAX ExT-CVC and Min ExT-NSIS are NP-hard since ExT-CVC and ExT-NSIS are NP-complete. Actually, for any class of graphs $\mathcal{G}$, Max ExT-CVC is NP-hard in $\mathcal{G}$ iff MiN ExT-NSIS is NP-hard in $\mathcal{G}$ since for any graph $G \in \mathcal{G}$ it can be shown that:

$$
o p t_{\mathrm{MAX} \text { Ext-CVC }}(G, U)+o p t_{\mathrm{MIN} \text { Ext-NSIS }}(G, V \backslash U)=|V| .
$$

The price of extension PoE is defined exactly as the ratio of approximation, i.e., the best possible lower (resp. upper) bound on $\frac{a p x}{o p t}$ that can be achieved in polynomial time. In particular, we say that Max ExT-CVC (resp. Min ExTNSIS) admits a polynomial $\rho$-PoE if for every instance $(G, U)$, we can efficiently compute a solution $S$ of $G$ which satisfies $|S \cap U| /$ opt $_{\text {Max Exт-CVC }}(G, U) \geqslant \rho$ (resp., $|S \cup U| / \operatorname{opt}_{\text {MiN ExT-NSIS }}(G, U) \leqslant \rho$ ).

Considering Max ExT-CVC on $G=(V, E)$ in the particular case $U=V$, we obtain a new problem called Upper Connected Vertex Cover (Upper CVC) where the goal is to find the largest minimal CVC. To our best knowledge, this problem has never been studied, although UPPER VC has been extensively studied $[5,14,25]$.

\section{UPPER CVC}

Input: A connected graph $G=(V, E)$.

Feasible Solution: Minimal connected vertex cover $S \subseteq V$.

Goal: Maximize $|S|$.

\subsection{Related work}

Garey and Johnson proved that (minimum) CVC is NP-hard in planar graphs of maximum degree 4 [16]. Moreover, it is shown in $[28,30]$ that the problem is polynomially solvable for graphs of maximum degree 3 , while NP-hardness proofs for bipartite and for bi-connected planar graphs of maximum degree 4 , are presented in $[12,15,26]$. The approximability of Min CVC has been considered in some more recent studies. The NP-hardness of approximating MiN CVC within $10 \sqrt{5}-21$ is proven in [15] while a 2-approximation algorithm is presented in [27]. Moreover, in [12] the problem is proven $A P X$-complete in 
bipartite graphs of maximum degree 4 . They also propose a $\frac{5}{3}$-approximation algorithm for MIN CVC for any class of graphs where MIN VC is polynomial-time solvable. Parameterized complexity for MIN CVC and MAX NSIS have been studied in $[23,24]$ while the enumeration of minimal connected vertex covers is investigated in [18] where it is shown that the number of minimal connected vertex covers of a graph of $n$ vertices is at most $1.8668^{n}$, and these sets can be enumerated in time $O\left(1.8668^{n}\right)$. For chordal graphs (even for chordality at most 5 ), the authors are able to give a better upper bound. The question to better understand the close relation between enumerations and extension problems is relevant because in this article we prove that ExT-CVC and MAX ExT-CVC are polynomial-time solvable in chordal graphs. Finally, one can find problems that are quite related to MIN CVC in [8].

Maximum minimal optimization variants have been studied for many classical graph problems in recent years, for example, in [5], Boria et al. have studied the Maximum Minimal Vertex Cover Problem (Upper VC in short) from the approximability and parameterized complexity point of views. The Minimum Maximal Independent Set problem, also called Minimum IndePEndent Dominating Set (Min ISDS) asks, given a graph $G=(V, E)$, for a subset $S \subseteq V$ of minimum size that is simultaneously independent and dominating. From the $N P$-hardness and exact solvability point of views, MIN IDS is equivalent to UPPER VC [25], but they seem to behave differently in terms of approximability and parameterized complexity [1]. Although MIN IDS is polynomially solvable in strongly chordal graphs [13], it is hard to approximate within $n^{(1-\epsilon)}$, for any $\epsilon>0$, in certain graph classes $[13,11]$. Regarding parameterized complexity, Fernau [14] presents an FPT-algorithm for UPPER VC with running time $\mathcal{O}^{*}\left(2^{k}\right)$, where $k$ is the size of an optimum solution, while it is proved that MIN IDS with respect to the standard parameter is $W[2]$-hard. Moreover, Boria et al. [5] provide a tight approximation result for UPPER VC in general graphs:

they present a $n^{\frac{1}{2}}$ approximation algorithm together with a proof that UPPER $\mathrm{VC}$ is $N P$-hard to approximate within $n^{\frac{1}{2}-\epsilon}$, for any $\epsilon>0$. Furthermore, they present a parameterized algorithm with running time $\left(1.5397^{k}\right)$ where $k$ is the standard parameter, by modifying the algorithm of [14]; they also show that weighted versions of UPPER VC and MIN IDS are in FPT with respect to the treewidth.

Regarding the extension variant of Dominating SET, namely ExT-DS, it is proven in $[22,21]$ that it is NP-complete, even in special graph classes like split graphs, chordal graphs, and line graphs. Moreover, a linear time algorithm for split graphs is given in [20] when $X, Y$ is a partition of the clique part. In [9], it is proved that ExT-VC is NP-complete in cubic graphs and in planar graphs of maximum degree 3 , while it is polynomially decidable in chordal and circular-arc graphs.

\subsection{Summary of results and organization}

The rest of the paper is organized as follows. In Section 2, after showing the relation between ExT-VC and ExT-CVC, we provide additional hardness results for 
ExT-CVC in bipartite graphs and weakly triangulated graphs, the latter leading to hardness results for UPPER VC and UPPER CVC. We then focus on bounds for PoE in Section 3, providing inapproximability results for MAX ExT-CVC in general and bipartite graphs. In Section 4 we discuss the (in)approximability of a special case of MAX EXT-CVC, namely UpPER CVC. Note that all results given in the paper for ExT-CVC are valid for ExT-NSIS as well. Due to lack of space the proofs of statements marked with $(*)$ are deferred to the full version of the paper.

\section{Solvability and hardness of extension problems}

Let us begin by some simple observations: $(G, U)$ with $G=(V, E)$ and $U \subseteq V$ is a yes-instance of ExT-CVC iff $(G, V \backslash U)$ is a yes-instance of ExT-NSIS. Hence, all complexity results given in this section for ExT-CVC are valid for ExT-NSIS as well. A leaf $\left(v \in V_{l}\right)$ never belongs to a minimal connected vertex cover $S$ (apart from the extreme case where $G$ consists of a single edge), while any cutvertex $v \in V_{c}$ necessarily belongs to $S$. This implies that for trees, we have a simple characterization of yes-instances for $n \geqslant 3:(T, U)$, where $T=(V, E)$ is a tree, is a yes-instance of ExT-CVC iff $U$ is a subset of cut-set $V_{c}$, or equivalently $U \subseteq V_{c}=V \backslash V_{l}$. For an edge or a cycle $C_{n}=(V, E),\left(C_{n}, U\right)$ is a yes-instance iff $U \neq V$; since a path $P_{n}=(V, E)$ is a special tree the case of graphs of maximum degree 2 is settled. Dealing with split graphs, a similar but more complicated characterization can be given. In the next subsection we will deduce more general results for ExT-CVC by showing and exploiting relations to ExT-VC.

\subsection{Relation between Ext-VC and Ext-CVC}

The following two properties allow to make use of known results for ExT-VC to obtain results for ExT-CVC.

Proposition 1. (*) ExT-CVC is polynomially reducible to EXT-VC in chordal graphs.

Proposition 2. ExT-CVC is NP-complete in graphs of maximum degree $\Delta+1$ if $\mathrm{ExT}-\mathrm{VC}$ is $\mathrm{NP}$-complete in graphs of maximum degree $\Delta$, and this holds even for bipartite graphs.

Proof. Given an instance $(G, U)$ of ExT-VC, where $G=(V, E)$ with $V=$ $\left\{v_{1}, \ldots, v_{n}\right\}$ and $U \subseteq V$, we build an instance $\left(G^{\prime}=\left(V^{\prime}, E^{\prime}\right), U^{\prime}\right)$ of ExT-CVC by adding a component $H=\left(V_{H}, E_{H}\right)$ to the original graph $G$.

The construction of $H$ is depicted to the right where $V_{H}=\left\{v_{i}^{\prime}, v_{i}^{\prime \prime}: 1 \leqslant i \leqslant n\right\}$ is the vertex set. The new instance of ExT-CVC is given by $\left(G^{\prime}, U^{\prime}\right)$ and consists of connecting the component $H$ to $G$ by linking $v_{i} v_{i}^{\prime}$ for each $1 \leqslant i \leqslant n$ and by setting $U^{\prime}=U$.

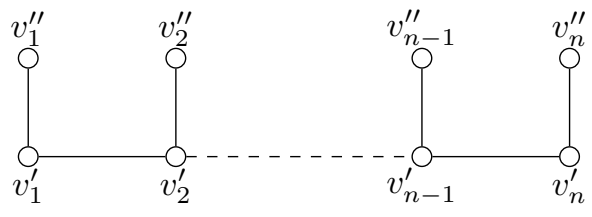


Clearly $G^{\prime}$ is of maximum degree $\Delta+1$ if $G$ is of maximum degree $\Delta$. Moreover, it is not difficult to see that $(G, U)$ is a yes-instance of ExT-VC iff $\left(G^{\prime}, U^{\prime}\right)$ is a yes-instance of ExT-CVC. To maintain bipartiteness, we apply an appropriate subdivision of $H$.

Using polynomial time decidability of ExT-VC in chordal graphs, parameterized complexity results (considering that the reduction increases the size of the instances only linearly), and NP-completeness in cubic bipartite graphs [9], we deduce:

Corollary 3. EXT-CVC is polynomial-time decidable in chordal graphs and $\mathrm{NP}$-complete in bipartite graphs of maximum degree 4. EXT-CVC parameterized with $|U|$ is $\mathrm{W}[1]$-complete, and there is no $2^{o(n+m)}$-algorithm for n-vertex, m-edge bipartite graphs of maximum degree 4 , unless ETH fails.

\subsection{Additional hardness results}

We first strengthen the hardness result of Corollary 3 to bipartite graphs of maximum degree 3 . This result could appear surprising since the optimization problem MiN CVC is polynomial-time solvable in graphs of maximum degree 3.

Theorem 4. EXT-CVC is NP-complete in bipartite graphs of maximum degree 3 even if $U$ is an independent set.

Proof. We reduce from 2-BALANCED 3-SAT, denoted (3, B2)-SAT, where an instance $I=(C, X)$ is given by a set $C$ of CNF clauses over a set of Boolean variables $X$ such that each clause has exactly 3 literals and each variable appears exactly 4 times, twice negative and twice positive. Deciding whether an instance of $(3, B 2)$-SAT is satisfiable is NP-complete by [4].

Consider an instance $(3, B 2)$-SAT with clauses $C=\left\{c_{1}, \ldots, c_{m}\right\}$ and variables $X=\left\{x_{1}, \ldots, x_{n}\right\}$. We build a bipartite graph $G=(V, E)$ together with a set of forced vertices $U$ as follows:

- For each clause $c=\ell_{1} \vee \ell_{2} \vee \ell_{3}$ where $\ell_{1}, \ell_{2}, \ell_{3}$ are literals, introduce a subgraph $H(c)=\left(V_{c}, E_{c}\right)$ with 6 vertices and 6 edges. $V_{c}$ contains three specified literal vertices $\ell_{c}^{1}, \ell_{c}^{2}, \ell_{c}^{3}$. The set of forced vertices in $H(c)$, denoted by $U_{c}$ is given by $U_{c}=\left\{\ell_{c}^{1}, \ell_{c}^{2}, \ell_{c}^{3}\right\}$. The gadget $H(c)$ is illustrated in the left part of Figure 1.

- For each variable $x$ introduce 21 new vertices which induce the subgraph $H(x)=\left(V_{x}, E_{x}\right)$ illustrated in Figure 1 . The vertex set $V_{x}$ contains four special vertices $t_{x}^{c_{1}}, t_{x}^{c_{2}}, f_{x}^{c_{3}}$ and $f_{x}^{c_{4}}$, where it is implicitly assumed (w.l.o.g.) that variable $x$ appears positively in clauses $c_{1}, c_{2}$ and negatively in clauses $c_{3}, c_{4}$. The independent set $U_{x}=\left\{1_{x}, 3_{x}, 5_{x}, 6_{x}, 8_{x}, 10_{x}, 12_{x}\right\}$ is in $U$ (i.e., forced to be in each feasible solution). The subgraph $H_{x}-U_{x}$ induced by $V_{x} \backslash U_{x}$ consists of an induced matching of size 5 and of 4 isolated vertices. 


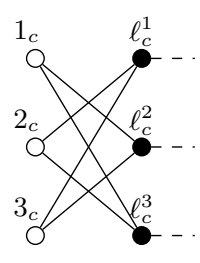

$H(c)$ for $c=\left(\ell_{1} \vee \ell_{2} \vee \ell_{3}\right)$
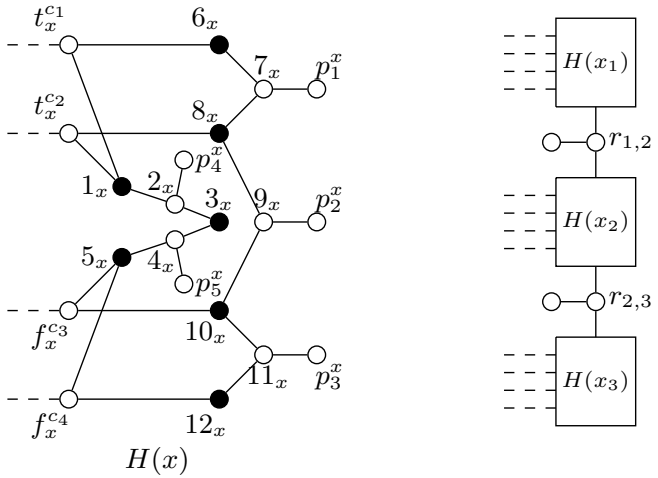

Fig. 1. Clause gadget $H(c)$ and Variable gadget $H(x)$ for ExT-CVC are shown on the left and in the middle of the figure respectively. Forced vertices (in $U$ ) are marked in Black. On the right, the way of connecting variable gadgets is depicted. Crossing edges between $H(c)$ and $H(x)$ are marked with dashed lines.

- We connect each gadget $H\left(x_{i}\right)$ to $H\left(x_{i+1}\right)$ by linking vertex $12_{x_{i}}$ to vertex $6_{x_{i+1}}$ using an intermediate vertex $r_{i, i+1}$ for all $1 \leqslant i \leqslant n-1$. We also add a pendant edge incident to each $r_{i, i+1}$ with leaf $r_{i, i+1}^{\prime}$; an illustration of this connection is depicted on the right of Figure 1.

- We interconnect $H(x)$ and $H(c)$ where $x$ is a variable occurring in literal $\ell_{i}$ of clause $c$ by adding edge $\ell_{c}^{i} t_{x}^{c}$ (resp., $\ell_{c}^{i} f_{x}^{c}$ ), where $t_{x}^{c}$ (resp., $f_{x}^{c}$ ) is in $H(x)$ and $\ell_{c}^{i}$ is in $H(c)$, if $x$ appears positively (resp., negatively) in clause $c$. These edges are called crossing edges.

Let $U=\left(\bigcup_{c \in C} U_{c}\right) \cup\left(\bigcup_{x \in X} U_{x}\right)$. This construction takes polynomial time and $G$ is a bipartite graph of maximum degree 3 .

Claim. $\left(^{*}\right) I=(C, X)$ is satisfiable iff $G$ admits a minimal CVC containing $U$.

The proof of the claim, deferred to the full version of the paper, completes the proof of the theorem.

Now, we will prove that the polynomial-time decidability of ExT-CVC in chordal graphs given in Corollary 3 cannot be extended to the slightly larger class of weakly chordal (also called weakly triangulated ${ }^{3}$ ) graphs which are contained in the class of 4-chordal graphs. For any integer $k \geqslant 3$, a graph is called $k$ chordal if it has no induced cycle of length greater than $k$. Thus, chordal graphs are precisely the 3 -chordal graphs. The problem of determining whether a graph is $k$-chordal is known to be co-NP-complete when $k$ is a part of the instance [29].

Theorem 5. (*) ExT-CVC is NP-complete in weakly triangulated graphs.

${ }^{3}$ This class is introduced in [19], as the class of graphs $G=(V, E)$ with no chordless cycle of five or more vertices in $G$ or in its complement $\bar{G}=(V, \bar{E})$. 


\section{Bounds on the price of extension of Max Ext-CVC}

Using Propositions 1 and 2, we can derive negative and positive approximation results for MAX ExT-CVC.

First, let us observe Min ExT-NSIS does not admit $O\left(n^{1-\varepsilon}\right)$-PoE even in the simplest case $U=\emptyset$ because there is a simple reduction from Min ISDS (also known as minimum maximal independent set $[11,13])$ to Min EXT-NSIS when $U=\emptyset$ by adding to the original graph $G=(V, E)$ two new vertices $\ell_{0}, \ell_{1}$ and edges $\ell_{0} \ell_{1}$ together with $\ell_{1} v$ for $v \in V$ (so, $\ell_{1}$ is an universal vertex); $\ell_{1}$ never belongs to a NSIS (or equivalently $\ell_{0}$ is a part of all maximal NSIS) because otherwise $\ell_{0}$ will become isolated. For general graphs, the price of extension associated to MAX ExT-CVC is one of the hardest problems to approximate.

Theorem 6. $(*)$ For any constant $\varepsilon>0$ and any $\rho \in \Omega\left(\frac{1}{\Delta^{1-\varepsilon}}\right)$ and $\rho \in$ $\Omega\left(\frac{1}{n^{1-\varepsilon}}\right)$, MAX EXT-CVC does not admit a polynomial $\rho$-PoE for general graphs of $n$ vertices and maximum degree $\Delta$, unless $\mathrm{P}=\mathrm{NP}$.

Although Proposition 2 preserves bipartiteness, we cannot immediately conclude the same kind of results since in [9] it is proved that MAX ExT-VC admits a polynomial $\frac{1}{2}-\mathrm{PoE}$ for bipartite graphs.

Theorem 7. $(*)$ For any constant $\varepsilon>0$ and any $\rho \in \Omega\left(\frac{1}{n^{1 / 2-\varepsilon}}\right)$, MAX EXT$\mathrm{CVC}$ does not admit a polynomial $\rho-\mathrm{PoE}$ for bipartite graphs of $n$ vertices, unless $\mathrm{P}=\mathrm{NP}$.

We next present a positive result, showing that the price of extension is equal to 1 in chordal graphs.

Proposition 8. (*) MAX EXT-CVC is polynomial-time solvable in chordal graphs.

\section{Approximability of UPPER CVC}

UpPer CVC is a special case of Max ExT-CVC where $U=V$. Regarding the approximability of UPPER CVC, we first show that an adaptation of Theorem 7 allows us to derive:

Corollary 9. $(*)$ For any constant $\varepsilon>0$, unless NP $=\mathrm{P}$, UpPen CVC is not $\Omega\left(\frac{1}{n^{1 / 3-\varepsilon}}\right)$-approximable in polynomial time for bipartite graphs on $n$ vertices.

On the positive side, we show that any minimal CVC is a $\frac{2}{\Delta(G)}$ approximation for UPPER CVC. To do this, we first give a structural property that holds for any minimal connected vertex cover. For a given connected graph $G=(V, E)$ let $S^{\star}$ be an optimal solution of UpPer CVC and $S$ be a minimal connected vertex cover of $G$. Denote by $A^{\star}=S^{\star} \backslash S$ and $A=S \backslash S^{\star}$ the proper parts of $S^{\star}$ and $S$ respectively, while $B=S \cap S^{\star}$ is the common part. Finally, $R=V \backslash\left(S^{\star} \cup S\right)$ denotes the rest of vertices. Also, for $X=A^{\star}$ or $X=A$, we set $X_{c}=\{v \in$ $\left.X: N_{G}(v) \subseteq B\right\}$ which is exactly the vertices of $X$ not having a neighbor in $\left(S \cup S^{\star}\right) \backslash X$. Actually, $\left(S \cup S^{\star}\right) \backslash X$ is either $S$ or $S^{\star}$. 
Lemma 10. (*) The following properties hold:

(i) For $X=A^{\star}$ or $X=A, X \cup R$ is an independent set of $G, G[X \cup B]$ is connected and $X_{c}$ is a subset of cut-set of $G[X \cup B]$.

(ii) Set $B$ is a dominating set of $G$.

The following theorem describes an interesting graph theoretic property. It relates the size of an arbitrary minimal connected vertex cover of a (connected) graph to the size of the largest minimal connected vertex cover.

Theorem 11. Any minimal CVC of a connected graph $G$ is a $\frac{2}{\Delta(G)}$-approximation for UPPER CVC.

Proof. Let $G=(V, E)$ be a connected graph. Let $S$ and $S^{\star}$ be a minimal CVC and an optimal one for UPPER CVC, respectively, and w.l.o.g., assume $|S|<$ $\left|S^{\star}\right|$. We prove the following inequalities:

$$
\left|A^{\star}\right| \leqslant(\Delta(G)-1)|B| \quad \text { and } \quad\left|A^{\star}\right| \leqslant(\Delta(G)-1)|A|
$$

Let us prove the first part $\left|A^{\star}\right| \leqslant(\Delta(G)-1)|B|$ of inequality (2). Consider $v_{1} \in B$ maximizing its number of neighbors in $A^{\star}$, i.e. $v_{1}=\arg \max \left\{\left|N_{A^{\star}}(v)\right|: v \in B\right\}$. Since $S$ is a minimal CVC with $|S|<\left|S^{\star}\right|$, we have $\Delta(G) \geqslant\left|N_{A^{\star}}\left(v_{1}\right)\right|+1$ from $(i)$ of Lemma 10 (otherwise $B=\left\{v_{1}\right\}$ with $d_{G}\left(v_{1}\right)=\Delta(G)$ ). In addition, from (ii) of Lemma 10 we have $N_{A^{\star}}(B)=A^{\star}$ and then $\sum_{v \in B}\left|N_{A^{\star}}(v)\right| \geqslant\left|N_{A^{\star}}(B)\right|=\left|A^{\star}\right|$. Putting together these inequalities we get $\left|A^{\star}\right| \leqslant|B|(\Delta(G)-1)$.

Let us prove the second part $\left|A^{\star}\right| \leqslant(\Delta(G)-1)|A|$ of inequality (2) using the following Claim:

Claim. $(*)$ There are at least $\left|A_{c}^{\star}\right|+|A|$ edges between $A$ and $B$ in $G[S]$.

Each vertex in $A^{\star} \backslash A_{c}^{\star}$ has by definition at least one neighbor in $A$, so we deduce: $\sum_{v \in A}|N(v)| \geqslant\left|A^{\star} \backslash A_{c}^{\star}\right|+|A|+\left|A_{c}^{\star}\right|=|A|+\left|A^{\star}\right|$. Now, by setting $a_{1}=\arg \max \left\{\left|N_{G}(v)\right|: v \in A\right\}$, we obviously get $|A|\left|N\left(a_{1}\right)\right| \geqslant \sum_{v \in A}|N(v)|$. Putting together these inequalities, we obtain: $|A| \Delta(G) \geqslant|A|\left|N\left(a_{1}\right)\right| \geqslant\left|A^{\star}\right|+|A|$ which leads to $\left|A^{\star}\right| \leqslant(\Delta(G)-1)|A|$. The inequality $|S| \geqslant \frac{2}{\Delta(G)}$ follows by considering the two cases $|A| \geqslant|B|$ and $|A|<|B|$.

A tight example of Theorem 11 for any $\Delta(G) \geqslant 3$ is illustrated to the right. The optimal solution for UPPER CVC contains $\Delta(G)$ vertices $\{a\} \cup\left\{v_{1}, \ldots, v_{\Delta(G)-1}\right\}$ while $\{a, b\}$ is a minimal connected vertex cover of size 2 .

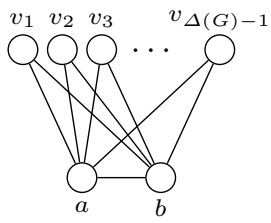




\section{References}

1. Giorgio Ausiello, Pierluigi Crescenzi, Giorgio Gambosi, Viggo Kann, Alberto Marchetti-Spaccamela, and Marco Protasi. Complexity and approximation: Combinatorial optimization problems and their approximability properties. Springer Science \& Business Media, 2012.

2. Cristina Bazgan, Ljiljana Brankovic, Katrin Casel, and Henning Fernau. On the complexity landscape of the domination chain. In Sathish Govindarajan and Anil Maheshwari, editors, Algorithms and Discrete Applied Mathematics - Second International Conference, CALDAM 2016, Thiruvananthapuram, India, February 1820, 2016, Proceedings, volume 9602 of Lecture Notes in Computer Science, pages 61-72. Springer, 2016.

3. Cristina Bazgan, Ljiljana Brankovic, Katrin Casel, Henning Fernau, Klaus Jansen, Kim-Manuel Klein, Michael Lampis, Mathieu Liedloff, Jérôme Monnot, and Vangelis Th. Paschos. The many facets of upper domination. Theor. Comput. Sci., $717: 2-25,2018$.

4. Piotr Berman, Marek Karpinski, and Alex D. Scott. Approximation hardness of short symmetric instances of MAX-3SAT. Electronic Colloquium on Computational Complexity (ECCC), (049), 2003.

5. Nicolas Boria, Federico Della Croce, and Vangelis Th Paschos. On the max min vertex cover problem. Discrete Applied Mathematics, 196:62-71, 2015.

6. Endre Boros, Vladimir Gurvich, and Peter L. Hammer. Dual subimplicants of positive Boolean functions. Optimization Methods and Software, 10(2):147-156, 1998.

7. Jean Cardinal and Eythan Levy. Connected vertex covers in dense graphs. Theor. Comput. Sci., 411(26-28):2581-2590, 2010.

8. Katrin Casel, Jan Dreier, Henning Fernau, Moritz Gobbert, Philipp Kuinke, Fernando Snchez Villaamil, Markus L. Schmid, and Erik Jan van Leeuwen. Complexity of independency and cliquy trees. Discrete Applied Mathematics (in press), 2019.

9. Katrin Casel, Henning Fernau, Mehdi Khosravian Ghadikolaei, Jérôme Monnot, and Florian Sikora. Extension of vertex cover and independent set in some classes of graphs and generalizations. CIAC 2019, LNCS (accepted), see also CoRR, abs/1810.04629, 2018.

10. Katrin Casel, Henning Fernau, Mehdi Khosravian Ghadikolaei, Jérôme Monnot, and Florian Sikora. On the complexity of solution extension of optimization problems. CoRR, abs/1810.04553, 2018.

11. Mirela Damian-Iordache and Sriram V Pemmaraju. Hardness of approximating independent domination in circle graphs. In International Symposium on Algorithms and Computation, pages 56-69. Springer, 1999.

12. Bruno Escoffier, Laurent Gourvès, and Jérôme Monnot. Complexity and approximation results for the connected vertex cover problem in graphs and hypergraphs. Journal of Discrete Algorithms, 8(1):36-49, 2010.

13. Martin Farber. Domination, independent domination, and duality in strongly chordal graphs. Discrete Applied Mathematics, 7(2):115-130, 1984.

14. Henning Fernau. Parameterized algorithmics: A graph-theoretic approach. Germany: Habilitationsschrift, Universität Tübingen, 2005.

15. Henning Fernau and David F Manlove. Vertex and edge covers with clustering properties: Complexity and algorithms. Journal of Discrete Algorithms, 7(2):149$167,2009$.

16. Michael R Garey and David S. Johnson. The rectilinear steiner tree problem is np-complete. SIAM Journal on Applied Mathematics, 32(4):826-834, 1977. 
17. Michael R. Garey and David S. Johnson. Computers and Intractability: A Guide to the Theory of NP-Completeness. W. H. Freeman \& Co., New York, NY, USA, 1979.

18. Petr A. Golovach, Pinar Heggernes, and Dieter Kratsch. Enumeration and maximum number of minimal connected vertex covers in graphs. European Journal of Combinatorics, 68:132 - 147, 2018. Combinatorial Algorithms, Dedicated to the Memory of Mirka Miller.

19. Ryan B. Hayward. Weakly triangulated graphs. J. Comb. Theory, Ser. B, 39(3):200-208, 1985.

20. Mamadou Moustapha Kanté, Vincent Limouzy, Arnaud Mary, and Lhouari Nourine. On the enumeration of minimal dominating sets and related notions. SIAM J. Discrete Math., 28(4):1916-1929, 2014.

21. Mamadou Moustapha Kanté, Vincent Limouzy, Arnaud Mary, Lhouari Nourine, and Takeaki Uno. Polynomial delay algorithm for listing minimal edge dominating sets in graphs. In Frank Dehne, Jörg-Rüdiger Sack, and Ulrike Stege, editors, Workshop on Algorithms and Data Structures, WADS, volume 9214 of lncs, pages 446-457. Springer, 2015.

22. Mamadou Moustapha Kanté, Vincent Limouzy, Arnaud Mary, Lhouari Nourine, and Takeaki Uno. A polynomial delay algorithm for enumerating minimal dominating sets in chordal graphs. In Ernst W. Mayr, editor, International Workshop on Graph-Theoretic Concepts in Computer Science, WG 2015, volume 9224 of lncs, pages 138-153. Springer, 2016.

23. Lukasz Kowalik and Marcin Mucha. A 9k kernel for nonseparating independent set in planar graphs. Theor. Comput. Sci., 516:86-95, 2014.

24. R. Krithika, Diptapriyo Majumdar, and Venkatesh Raman. Revisiting connected vertex cover: FPT algorithms and lossy kernels. Theory Comput. Syst., 62(8):1690$1714,2018$.

25. David F Manlove. On the algorithmic complexity of twelve covering and independence parameters of graphs. Discrete Applied Mathematics, 91(1-3):155-175, 1999.

26. PK Priyadarsini and T Hemalatha. Connected vertex cover in 2-connected planar graph with maximum degree 4 is NP-complete. International Journal of Mathematical, Physical and Engineering Sciences, 2(1):51-54, 2008.

27. Carla Savage. Depth-first search and the vertex cover problem. Information Processing Letters, 14(5):233-235, 1982.

28. Ewald Speckenmeyer. On feedback vertex sets and nonseparating independent sets in cubic graphs. Journal of Graph Theory, 12(3):405-412, 1988.

29. Ryuhei Uehara. Tractable and intractable problems on generalized chordal graphs. Technical report, Technical Report COMP98-83, IEICE, 1999.

30. Shuichi Ueno, Yoji Kajitani, and Shin'ya Gotoh. On the nonseparating independent set problem and feedback set problem for graphs with no vertex degree exceeding three. Discrete Mathematics, 72(1-3):355-360, 1988. 